\title{
Circuit Design of Surface Acoustic Wave Based Micro Force Sensor
}

\author{
Yuanyuan Li, ${ }^{1,2}$ Wenke Lu, ${ }^{1}$ Changchun Zhu, ${ }^{3}$ Qinghong Liu, ${ }^{4}$ \\ Haoxin Zhang, ${ }^{4}$ and Chenchao Tang ${ }^{2}$ \\ ${ }^{1}$ College of Information Science and Technology, Donghua University, Shanghai 201620, China \\ ${ }^{2}$ College of Electronic and Electrical Engineering, Shanghai University of Engineering Science, Shanghai 201620, China \\ ${ }^{3}$ College of Electronics and Information Engineering, Xian Jiaotong University, Xian 710049, China \\ ${ }^{4}$ Xian Leitong Science \& Technology Co. Ltd., Xian 710049, China
}

Correspondence should be addressed to Wenke Lu; luwenke3@163.com

Received 10 February 2014; Revised 7 April 2014; Accepted 7 April 2014; Published 29 April 2014

Academic Editor: Weichao Sun

Copyright (C) 2014 Yuanyuan Li et al. This is an open access article distributed under the Creative Commons Attribution License, which permits unrestricted use, distribution, and reproduction in any medium, provided the original work is properly cited.

Pressure sensors are commonly used in industrial production and mechanical system. However, resistance strain, piezoresistive sensor, and ceramic capacitive pressure sensors possess limitations, especially in micro force measurement. A surface acoustic wave (SAW) based micro force sensor is designed in this paper, which is based on the theories of wavelet transform, SAW detection, and pierce oscillator circuits. Using lithium niobate as the basal material, a mathematical model is established to analyze the frequency, and a peripheral circuit is designed to measure the micro force. The SAW based micro force sensor is tested to show the reasonable design of detection circuit and the stability of frequency and amplitude.

\section{Introduction}

Wavelet transform finds its application in many disciplines and fields such as in image processing, water-sound, earthquake detection, biomedicine, mechanical vibration, pronunciation recognition, communication, chemical industry, and torrent analysis [1]. SAW devices are early examples of microelectromechanical systems (MEMS) because of the coupling needed between the electrical and mechanical properties as discussed by Ballantine et al. [2]. The method of implementing wavelet transform with SAW devices has been first proposed by Peng et al. [3-5]. The wavelet transform device of SAW can benefit from the excellent properties of the SAW devices, namely, passive, small size, low cost, excellent temperature stability, high reliability, and high reproducibility, which overcomes the complicated algorithms and high power for VLSI $[6,7]$, and big size and low reproducibility for optical devices [8]. Wave propagation along the surface allows the sensitivity of the wavelet transform device of SAW to change in the external environment and the development of these sensors for applications such as gas detection, changes in fluid viscosity, determination of stiffness constants of mechanical vibration, and detection of the onset of ice formation on aerospace structures [9-11].

Nowadays, micromanipulation has performed to design either mobile micro robots or a precise positioning device under the control of mechanical systems. Various tools for manipulating micro parts and assembling micro systems have been developed and integrated. Semiconductor strain gauges are preferred when small forces have been measured. M. Jungwirth has described the micromechanical precision pressure sensor but in delay lines. A silicon based micro force sensor has been developed with larger electromechanical coupling coefficient $k 2$. The three-axis micro force sensor has been designed by Jungwirth et al. with the problem of measurement uncertainty. The dual axis micro force sensor for robotic manipulations needs to use strain gauges [12-15].

This paper proposes to use substrate materials of small electromechanical coupling coefficient $k 2\left(128^{\circ} Y\right.$ shear $\mathrm{LiNbO}_{3}$ ) in the manufacture to design the wavelet transform device of SAW based micro force sensor, which has high accuracy and sensitivity testing precision. In addition, 
a mathematical model is established to analyze the frequency of our sensor and a peripheral detection circuit is designed. Within the scope of effective measurement, the SAW based micro force sensor possesses good linearity, consistency, and repeatability in performance. Besides, it uses the piezoelectric properties and the temperature stability of the crystal, as well as the frequency signal instead of the conventional pressure sensor with voltage signal, which makes the signal processing of this device more digital and possesses more stable performances.

The succeeding sections are organized as follows. Section 2 introduces the fundamental principles of designing interdigital transducers (IDT) for wavelet transform device of SAW. In Section 3, the peripheral detection circuit is explained. In addition, the linear regression model is provided in Section 4. Finally Section 5 delivers the testing and analysis of SAW based micro force sensor which is used for measuring various micro force.

\section{Design IDT for Wavelet Transform Device of SAW}

The wavelet function is

$$
\psi_{s}(t)=\frac{1}{\sqrt{s}} \psi\left(\frac{t}{s}\right)
$$

where $s$ denotes the scale of wavelet function.

The wavelet transform of signal $f(t)$ is

$$
\begin{aligned}
\mathrm{WT}_{s}(\tau) & =f(t) * \psi_{s}(t) \\
& =\int_{R} f(t) \frac{1}{\sqrt{s}} \psi\left(\frac{\tau-t}{s}\right) d t \\
& =\frac{1}{\sqrt{s}} \int_{R} f(t) \psi\left(\frac{\tau-t}{s}\right) d t .
\end{aligned}
$$

When $\psi_{s}(t)$ is a Morlet wavelet function, formula (1) is converted into [3-5]

$$
\psi_{s}(t)=\frac{1}{\sqrt{s}} e^{-(1 / 2)(t / s)^{2}} e^{j 2 \pi\left(f_{0} / s\right) t}=P_{s}(t) e^{j 2 \pi\left(f_{0} / s\right) t},
$$

where $P_{s}(t)$ is the wavelet-envelope function, $P_{s}(t)=$ $(1 / \sqrt{s}) e^{-1 / 2(t / s)^{2}}$, and $f_{0} / s$ is the center frequency. When $s=2 k, k$ is a random number from $-\infty$ to $+\infty$. The wavelet function shown in formula (3) is converted into the Morlet dyadic wavelet function $[4,5]$. The microwave communication equipment of this paper design needs a singlescale wavelet transform processor of the center frequency $f_{0}$, which can be rewritten as

$$
f_{0}=\frac{v_{s}}{\lambda}=\frac{v_{s}}{2(a+b)},
$$

where $\lambda=2(a+b)$ is the wavelength, $v_{s}$ is the speed of the SAW, and $a$ and $b$ are the width and the interval of IDT.

The delay line in wavelet transform device of SAW designed in this paper exhibits the basic structure in Figure 1 and two acoustic electric transducers on the piezoelectric characteristic substrate material polishing surface, named input IDT and output IDT.

As the Morlet wavelet transform of SAW devices is based on formula (3), once all the parameters in (4) are relatively fixed, its center frequency of each scale should be corresponding with the only device. The device has been designed and produced in this paper that is shown in Figure 2.

\section{Design of the Peripheral Detection Circuit}

3.1. The Principle of Peripheral Detection Circuit. After making the fabricated device, the peripheral detection circuit needs to be designed to get $F_{m}$ and $f$ which are the pressure and the output frequency of the SAW based micro force sensor. Figure 3 shows the general structure of the SAW micro force sensor with IDTs.

If the SAW resonator density of the piezoelectric substrate is $m$, the unit is $\mathrm{m} / \mathrm{g}^{2}$, the dielectric constant is $\varepsilon$, the electrical conductivity is $\sigma$, the elastic parameter is $c$, the unit is $\mathrm{n} / \mathrm{m}^{2}$, the environment temperature is $t$, and the pressure is $p$ [16], $v_{s}$ based on the theory of surface disturbance is given as

$$
v_{s}=v(m, \varepsilon, \sigma, c, t, p) \text {. }
$$

After derivation, (5) can be changed to

$$
\begin{array}{r}
\frac{\Delta v_{s}}{v_{s}}=\frac{1}{v_{s}}\left(\frac{\partial v}{\partial m} \Delta m+\frac{\partial v}{\partial \varepsilon} \Delta \varepsilon+\frac{\partial v}{\partial \sigma} \Delta \sigma\right. \\
\left.+\frac{\partial v}{\partial c} \Delta c+\frac{\partial v}{\partial t} \Delta t+\frac{\partial v}{\partial p} \Delta p\right) .
\end{array}
$$

Equation (6) has been used to calculate the relationship between the propagation speed of available and the SAW oscillator frequency which is equal to the following equation:

$$
\begin{aligned}
& \frac{\Delta f}{f_{0}}=\frac{\Delta v_{s}}{v_{s}} \\
&=-\frac{1}{v_{\text {SAW }}}\left(\frac{\partial v}{\partial m} \Delta m+\frac{\partial v}{\partial \varepsilon} \Delta \varepsilon+\frac{\partial v}{\partial \sigma} \Delta \sigma\right. \\
&\left.+\frac{\partial v}{\partial c} \Delta c-\frac{\partial v}{\partial t} \Delta t+\frac{\partial v}{\partial p} \Delta p\right) .
\end{aligned}
$$

The change in the oscillator frequency and the measurement of the parameters can be obtained by using (7), which is the working principle of the SAW micro force sensor circuit.

3.2. The Actual Oscillator Circuit. The mixing frequency circuit is divided into two oscillator circuits, one for the reference oscillator circuit with a fixed frequency, and the other for the detector circuit. The frequency difference can be received from the mixing circuit. Figure 4 shows the circuit framework.

The oscillator circuit with fixed frequency in the dualchannel structure has an output signal, which is used as the stable signal source during measurement. The digital signal processing circuit is composed of a filter, a mixer, an amplifier, and a shaping circuit. 


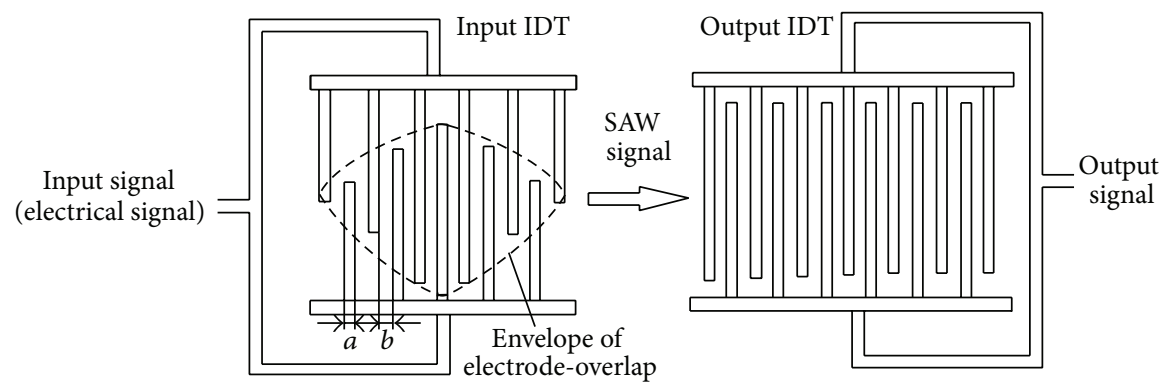

FIgURE 1: The transducer substrate of wavelet transform device of SAW.

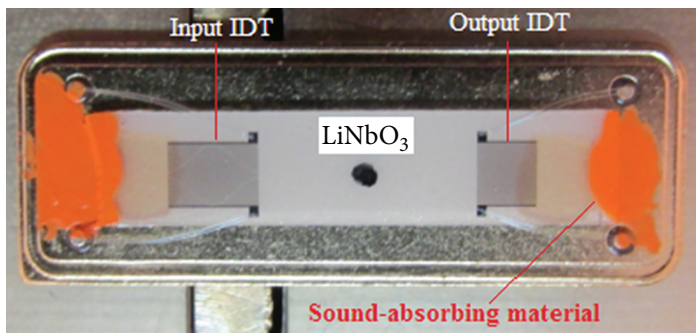

FIgURE 2: The fabricated single-scale wavelet transform device of SAW.

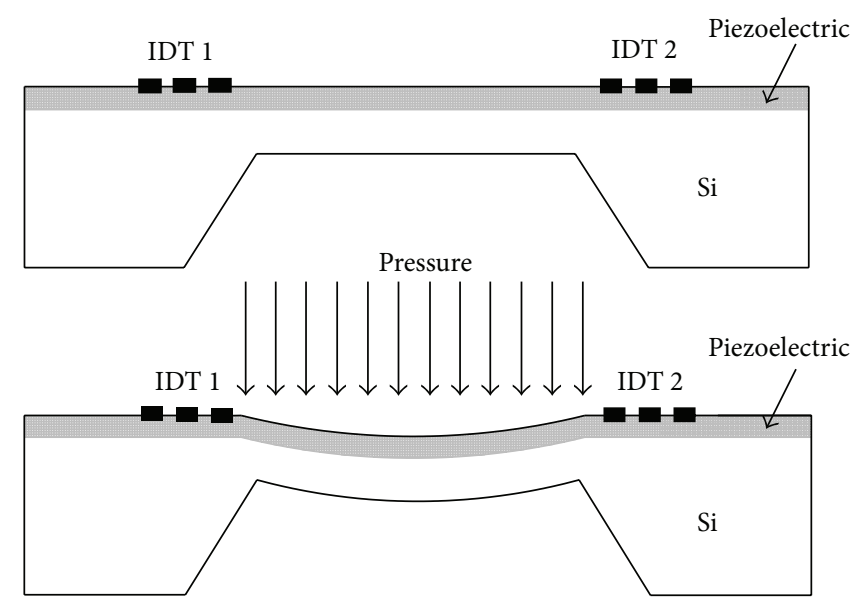

FIGURE 3: General structure of the SAW micro force sensor with two IDTs.

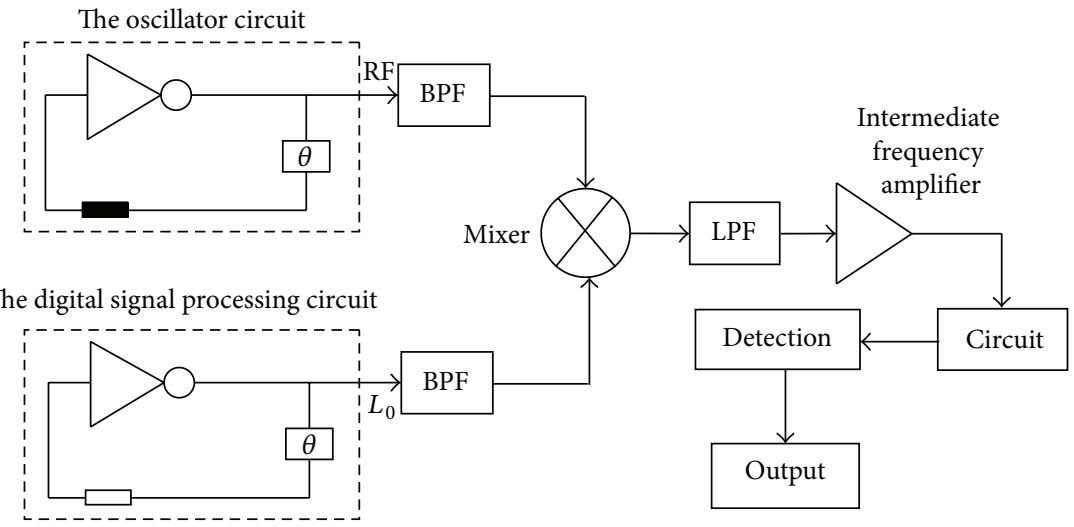

FIGURE 4: The circuit framework of fixed frequency circuit. 
For a surface acoustic generator as the sensitive element of the SAW micro force sensor, the frequency stability directly affects the resolution of the testing precision. The oscillator frequency has depended on the conditions of the feedback loop phase. Therefore, the improvement of the SAW oscillator frequency stability also improves the performance of the micro force sensor.

The Pierce oscillator circuit has a better stability compared with Colpitts and Clapp circuits [17]. Figure 5 shows the oscillator circuit of the SAW based micro force sensor based on the principle of Pierce circuit and the combination with the design requirements.

The high-frequency triode 2SC3357 affects the resonance amplifier in the circuit by ensuring the normal startup of the oscillator circuit. $R_{1}$ is the DC bias resistance of this triode, and adjusting the value changes with $\mathrm{DC}$ working points. $R_{2}$ is the DC bias resistance of the triode $s 8050$ that changes the DC working points and transforms the bias current of the circuit. This triode functions as the current source and stabilizes the circuit working state. The SAW resonator affects the frequency selection in the feedback loop by inducing the SAW oscillator frequency stability and improving the antijamming ability.

To ensure that the feedback loop circuit phase is in balance, the inductance $L$ has been added to the Pierce circuit to eliminate the effect of the stability of the DC voltage source from the oscillator circuit. The LC parallel resonant circuit also affects the DC bypass circuit. The feedback coefficient $F$ is related to $C_{1}$ and $C_{2}$ and is given as

$$
F=\frac{C_{1}}{C_{2}},
$$

where $C_{3}, C_{4}$, and $L_{2}$ are the decoupling devices of the DC source that can eliminate the effect of the oscillator circuit from the LC filter function.

3.3. Determine the Parameters. The circuit layout, selection of components, and calculated parameters are important. The work frequency of the micro force sensor is $50 \mathrm{MHz}$; the center frequency of the LC parallel resonant circuit should be

$$
f_{0}=\frac{1}{2 \pi \sqrt{\mathrm{LC}}} .
$$

To ensure that the amplitude of oscillator circuit has better characteristics than the initial conditions, $C_{2}$ should be smaller. In addition, the designed oscillator frequency is given; the capacitance is selected as $5.1 \mathrm{pF}$. From (10), $L$ can be obtained as

$$
L=\frac{1}{\left(2 \pi f_{0}\right)^{2} C}
$$

Because parasitic parameters in practical circuits affect their performances, $L$ must be adjustable. By changing the number of turns of the air-core coil, the coil inductance can be fine-tuned, and the frequency of the $L C$ parallel resonant circuit will be in accordance with the working frequency

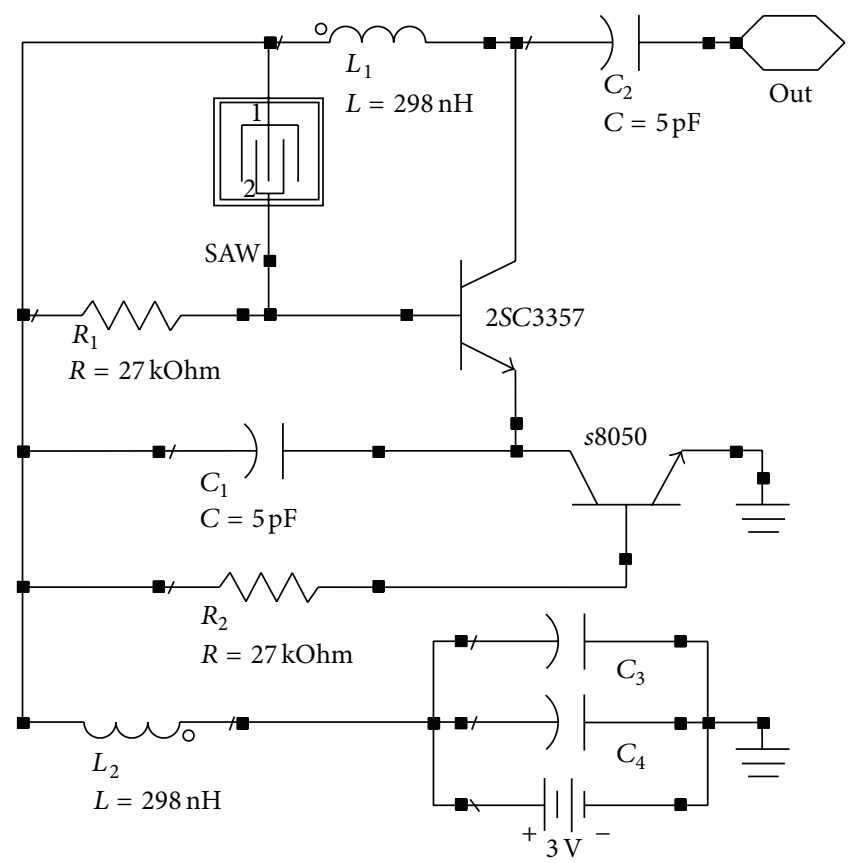

Figure 5: The Pierce circuit of the SAW based micro force sensor.

of the SAW based micro force sensor. The air-core coil inductance has been calculated by the following equation:

$$
L=\frac{0.01 \times D \times N^{2}}{\left(L_{N} / D\right)+0.44},
$$

where $L$ is the coil inductance (in $\mu \mathrm{H}$ ), $D$ is the diameter of the coil (in $\mathrm{cm}$ ), $N$ is the number of coil turns, and $L N$ is the coil length (in $\mathrm{cm}$ ).

An oscillator frequency source should have good stability, phase noise, and high $Q$ value [18]. Figure 6 shows the equivalent circuit, with $C_{2}$ expressed in picofarads.

The SAW resonator can work in series or parallel resonant frequencies upon operating in the feedback loop. The transistor 2SC3357 and $s 8050$ provide the DC bias current in the oscillator circuit design based on the requirements.

\section{The Linear Regression Model of the SAW Based Micro Force Sensor}

4.1. Establishment of the Linear Regression Model. After getting the pressure of SAW sensor $\left(F_{m}\right)$ and the output frequency $(f)$ from the actual oscillator circuit, the fitting function should be established. The relationship between the pressure of this sensor $\left(F_{m}\right)$ and the output frequency $(f)$ is given as follows:

$$
F_{m}=k_{0}+k_{1} \Delta f+k_{2} \Delta f^{2}+k_{3} \Delta f^{3}+\cdots+k_{n} \Delta f^{n} \cdots .
$$

If $F_{i}, f_{i}, i=1,2, \ldots, n$, and $n=5$, the method of least squares can be used to solve this function is given as

$$
\begin{aligned}
F_{m}= & k_{0}+k_{1} \Delta f+k_{2} \Delta f^{2}+k_{3} \Delta f^{3} \\
& +k_{4} \Delta f^{4}+k_{5} \Delta f^{5}+k_{6} \Delta f^{6} .
\end{aligned}
$$




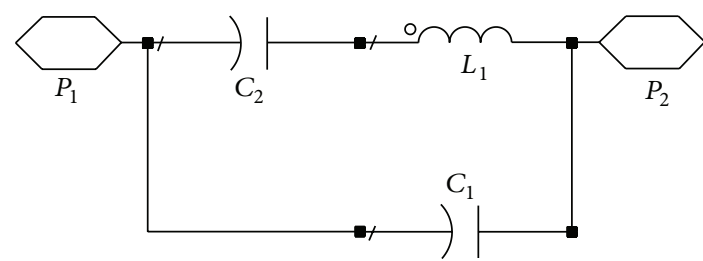

FIgUre 6: The equivalent circuit of the SAW device.

The regression coefficients $k_{0}, k_{1}, k_{2}, k_{3}, k_{4}, k_{5}$, and $k_{6}$ can be calculated through formula (13).

4.2. Calculate the Input and Output Variable Regression. The sum of the variance $(Q)$ between the dependent and independent variables is given as [19]

$$
Q\left(k_{0}-k_{6}\right)=\sum_{i=1}^{N} \Delta_{i}^{2}=\sum_{i=1}^{N}\left[\Delta f_{i}-F_{i}\right]^{2}
$$

where $N$ is the sampling point of the independent variables. Therefore, the least squares estimation calculates the minimum value of $Q$, which implies that $\hat{k}_{0}, \hat{k}_{1}, \hat{k}_{2}, \hat{k}_{3}, \hat{k}_{4}, \hat{k}_{5}$, and $k_{6}$ must suit the following equation:

$$
\begin{aligned}
& Q\left(\hat{k}_{0}, \hat{k}_{1}, \hat{k}_{2}, \hat{k}_{3}, \hat{k}_{4}, \hat{k}_{5}, \hat{k}_{6}\right) \\
& =\sum_{i=1}^{N}\left[\Delta f_{i}-F_{i}\right]^{2}=\min _{\substack{\hat{k}_{0}-\hat{k}_{6} \\
\sum_{i=1}}}^{N}\left[\Delta f_{i}-F_{i}\right]^{2},
\end{aligned}
$$

where $\hat{k}_{0}, \hat{k}_{1}, \hat{k}_{2}, \hat{k}_{3}, \hat{k}_{4}, \hat{k}_{5}$, and $k_{6}$ are the respective least squares estimations of $k_{0}, k_{1}, k_{2}, k_{3}, k_{4}, k_{5}$, and $k_{6}$.

When

$$
\begin{aligned}
& \left.\frac{\partial Q\left(k_{0}, k_{1}, k_{2}, k_{3}, k_{4}, k_{5}\right)}{\partial k_{0}}\right|_{k_{0}=\hat{k}_{0}}=0, \\
& \left.\frac{\partial Q\left(k_{0}, k_{1}, k_{2}, k_{3}, k_{4}, k_{5}\right)}{\partial k_{1}}\right|_{k_{0}=\hat{k}_{1}}=0 \text {, } \\
& \left.\frac{\partial Q\left(k_{0}, k_{1}, k_{2}, k_{3}, k_{4}, k_{5}\right)}{\partial k_{2}}\right|_{k_{0}=\hat{k}_{2}}=0 \text {, } \\
& \left.\frac{\partial Q\left(k_{0}, k_{1}, k_{2}, k_{3}, k_{4}, k_{5}\right)}{\partial k_{3}}\right|_{k_{0}=\hat{k}_{3}}=0 \text {, } \\
& \left.\frac{\partial Q\left(k_{0}, k_{1}, k_{2}, k_{3}, k_{4}, k_{5}\right)}{\partial k_{4}}\right|_{k_{0}=\hat{k}_{4}}=0 \text {, } \\
& \left.\frac{\partial Q\left(k_{0}, k_{1}, k_{2}, k_{3}, k_{4}, k_{5}\right)}{\partial k_{5}}\right|_{k_{0}=\hat{k}_{5}}=0 \text {, } \\
& \left.\frac{\partial Q\left(k_{0}, k_{1}, k_{2}, k_{3}, k_{4}, k_{5}\right)}{\partial k_{6}}\right|_{k_{0}=\hat{k}_{6}}=0 \text {. }
\end{aligned}
$$

The equation (17) can be derived by formula (15) and (16)

$$
\begin{aligned}
\sum_{i=1}^{N}\left[\left(\hat{k}_{0}\right.\right. & +\hat{k}_{1} \Delta f_{i}+\hat{k}_{2} \Delta f_{i}^{2}+\hat{k}_{3} \Delta f_{i}^{3} \\
& \left.\left.+\hat{k}_{4} \Delta f_{i}^{4}+\hat{k}_{5} \Delta f_{i}^{5}+\hat{k}_{6} \Delta f_{i}^{6}\right)-F_{i}\right]=0,
\end{aligned}
$$

$\sum_{i=1}^{N}\left[\left(\hat{k}_{0}+\hat{k}_{1} \Delta f_{i}+\hat{k}_{2} \Delta f_{i}^{2}+\hat{k}_{3} \Delta f_{i}^{3}\right.\right.$

$$
\left.\left.+\hat{k}_{4} \Delta f_{i}^{4}+\hat{k}_{5} \Delta f_{i}^{5}+\hat{k}_{6} \Delta f_{i}^{6}\right)-F_{i}\right] \Delta f_{i}=0,
$$

$\sum_{i=1}^{N}\left[\left(\hat{k}_{0}+\hat{k}_{1} \Delta f_{i}+\hat{k}_{2} \Delta f_{i}^{2}+\hat{k}_{3} \Delta f_{i}^{3}\right.\right.$

$$
\left.\left.+\hat{k}_{4} \Delta f_{i}^{4}+\hat{k}_{5} \Delta f_{i}^{5}+\hat{k}_{6} \Delta f_{i}^{6}\right)-F_{i}\right] \Delta f_{i}^{2}=0
$$

$\sum_{i=1}^{N}\left[\left(\hat{k}_{0}+\hat{k}_{1} \Delta f_{i}+\hat{k}_{2} \Delta f_{i}^{2}+\hat{k}_{3} \Delta f_{i}^{3}\right.\right.$

$$
\left.\left.+\hat{k}_{4} \Delta f_{i}^{4}+\hat{k}_{5} \Delta f_{i}^{5}+\hat{k}_{6} \Delta f_{i}^{6}\right)-F_{i}\right] \Delta f_{i}^{3}=0
$$

$$
\begin{aligned}
\sum_{i=1}^{N}\left[\left(\hat{k}_{0}\right.\right. & +\hat{k}_{1} \Delta f_{i}+\hat{k}_{2} \Delta f_{i}^{2}+\hat{k}_{3} \Delta f_{i}^{3} \\
& \left.\left.+\hat{k}_{4} \Delta f_{i}^{4}+\hat{k}_{5} \Delta f_{i}^{5}+\hat{k}_{6} \Delta f_{i}^{6}\right)-F_{i}\right] \Delta f_{i}^{4}=0,
\end{aligned}
$$

$\sum_{i=1}^{N}\left[\left(\hat{k}_{0}+\hat{k}_{1} \Delta f_{i}+\hat{k}_{2} \Delta f_{i}^{2}+\hat{k}_{3} \Delta f_{i}^{3}\right.\right.$

$$
\begin{aligned}
&\left.\left.+\hat{k}_{4} \Delta f_{i}^{4}+\hat{k}_{5} \Delta f_{i}^{5}+\hat{k}_{6} \Delta f_{i}^{6}\right)-F_{i}\right] \Delta f_{i}^{5}=0, \\
& \sum_{i=1}^{N}\left[\left(\hat{k}_{0}+\hat{k}_{1} \Delta f_{i}+\hat{k}_{2} \Delta f_{i}^{2}+\hat{k}_{3} \Delta f_{i}^{3}\right.\right. \\
&\left.\left.+\hat{k}_{4} \Delta f_{i}^{4}+\hat{k}_{5} \Delta f_{i}^{5}+\hat{k}_{6} \Delta f_{i}^{6}\right)-F_{i}\right] \Delta f_{i}^{6}=0 .
\end{aligned}
$$

Equation (18) can be derived from formulas (14) and (17). Consider

$$
\begin{aligned}
& N \hat{k}_{0}+\hat{k}_{1} \sum_{i=1}^{N} \Delta f+\hat{k}_{2} \sum_{i=1}^{N} \Delta f^{2}+\hat{k}_{3} \sum_{i=1}^{N} \Delta f^{3} \\
&+\hat{k}_{4} \sum_{i=1}^{N} \Delta f^{4}+\hat{k}_{5} \sum_{i=1}^{N} \Delta f^{5}+\hat{k}_{6} \sum_{i=1}^{N} \Delta f^{6}=\sum_{i=1}^{N} F_{i}, \\
& \hat{k}_{0} \sum_{i=1}^{N} \Delta f+\hat{k}_{1} \sum_{i=1}^{N} \Delta f^{2}+\hat{k}_{2} \sum_{i=1}^{N} \Delta f^{3}+\hat{k}_{3} \sum_{i=1}^{N} \Delta f^{4} \\
&+\hat{k}_{4} \sum_{i=1}^{N} \Delta f^{5}+\hat{k}_{5} \sum_{i=1}^{N} \Delta f^{6}+\hat{k}_{6} \sum_{i=1}^{N} \Delta f^{7}=\sum_{i=1}^{N} F_{i} \Delta f_{i},
\end{aligned}
$$




$$
\begin{gathered}
\hat{k}_{0} \sum_{i=1}^{N} \Delta f^{2}+\hat{k}_{1} \sum_{i=1}^{N} \Delta f^{3}+\hat{k}_{2} \sum_{i=1}^{N} \Delta f^{4}+\hat{k}_{3} \sum_{i=1}^{N} \Delta f^{5} \\
+\hat{k}_{4} \sum_{i=1}^{N} \Delta f^{6}+\hat{k}_{5} \sum_{i=1}^{N} \Delta f^{7}+\hat{k}_{6} \sum_{i=1}^{N} \Delta f^{8}=\sum_{i=1}^{N} F_{i} \Delta f_{i}^{2}, \\
\hat{k}_{0} \sum_{i=1}^{N} \Delta f^{3}+\hat{k}_{1} \sum_{i=1}^{N} \Delta f^{4}+\hat{k}_{2} \sum_{i=1}^{N} \Delta f^{5}+\hat{k}_{3} \sum_{i=1}^{N} \Delta f^{6} \\
+\hat{k}_{4} \sum_{i=1}^{N} \Delta f^{7}+\hat{k}_{5} \sum_{i=1}^{N} \Delta f^{8}+\hat{k}_{6} \sum_{i=1}^{N} \Delta f^{9}=\sum_{i=1}^{N} F_{i} \Delta f_{i}^{3}, \\
\hat{k}_{0} \sum_{i=1}^{N} \Delta f^{4}+\hat{k}_{1} \sum_{i=1}^{N} \Delta f^{5}+\hat{k}_{2} \sum_{i=1}^{N} \Delta f^{6}+\hat{k}_{3} \sum_{i=1}^{N} \Delta f^{7} \\
+\hat{k}_{4} \sum_{i=1}^{N} \Delta f^{10}+\hat{k}_{5} \sum_{i=1}^{N} \Delta f^{11}+\hat{k}_{6} \sum_{i=1}^{N} \Delta f^{12}=\sum_{i=1}^{N} F_{i} \Delta f_{i}^{6} \\
+\hat{k}_{4} \sum_{i=1}^{N} \Delta f^{8}+\hat{k}_{5} \sum_{i=1}^{N} \Delta f^{9}+\hat{k}_{6} \sum_{i=1}^{N} \Delta f^{10}=\sum_{i=1}^{N} F_{i} \Delta f_{i}^{4}, \\
\hat{k}_{0} \sum_{i=1}^{N} \Delta f^{5}+\hat{k}_{1} \sum_{i=1}^{N} \Delta f^{9}+\hat{k}_{5} \sum_{i=1}^{N} \Delta f^{10}+\hat{k}_{6} \sum_{i=1}^{N} \Delta f^{11}=\sum_{i=1}^{N} F_{i} \Delta f_{i}^{5}, \\
\hat{k}_{i=1} \hat{k}^{7}+\hat{k}_{3} \sum_{i=1}^{N} \Delta f^{8}
\end{gathered}
$$

Equation (18) can be written as

$$
\begin{aligned}
& N \hat{k}_{0}+A \hat{k}_{1}+B \hat{k}_{2}+C \hat{k}_{3}+D \hat{k}_{4}+E \hat{k}_{5}+F \hat{k}_{6}=M, \\
& A \hat{k}_{0}+B \hat{k}_{1}+C \hat{k}_{2}+D \hat{k}_{3}+E \hat{k}_{4}+F \hat{k}_{5}+G \hat{k}_{6}=P, \\
& \hat{k}_{0}+C \hat{k}_{1}+D \hat{k}_{2}+E \hat{k}_{3}+F \hat{k}_{4}+G \hat{k}_{5}+H \hat{k}_{6}=Q, \\
& C \hat{k}_{0}+D \hat{k}_{1}+E \hat{k}_{2}+F \hat{k}_{3}+G \hat{k}_{4}+H \hat{k}_{5}+I \hat{k}_{6}=R, \\
& \wedge \hat{k}_{0}+E \hat{k}_{1}+F \hat{k}_{2}+G \hat{k}_{3}+H \hat{k}_{4}+I \hat{k}_{5}+J \hat{k}_{6}=S, \\
& E \hat{k}_{0}+F \hat{k}_{1}+G \hat{k}_{2}+H \hat{k}_{3}+I \hat{k}_{4}+J \hat{k}_{5}+K \hat{k}_{6}=T, \\
& \hat{k}_{0}+G \hat{k}_{1}+H \hat{k}_{2}+I \hat{k}_{3}+J \hat{k}_{4}+K \hat{k}_{5}+L \hat{k}_{6}=U,
\end{aligned}
$$

where

$$
\begin{aligned}
& A=\sum_{i=1}^{N} \Delta f_{i}, \quad B=\sum_{i=1}^{N} \Delta f_{i}^{2}, \\
& C=\sum_{i=1}^{N} \Delta f_{i}^{3}, \quad D=\sum_{i=1}^{N} \Delta f_{i}^{4}, \\
& E=\sum_{i=1}^{N} \Delta f_{i}^{5}, \quad F=\sum_{i=1}^{N} \Delta f_{i}^{6}, \\
& G=\sum_{i=1}^{N} \Delta f_{i}^{7} \\
& H=\sum_{i=1}^{N} \Delta f_{i}^{8}, \quad I=\sum_{i=1}^{N} \Delta f_{i}^{9}, \\
& J=\sum_{i=1}^{N} \Delta f_{i}^{10}, \quad K=\sum_{i=1}^{N} \Delta f_{i}^{11}, \\
& L=\sum_{i=1}^{N} \Delta f_{i}^{12} \\
& M=\sum_{i=1}^{N} F_{i}, \quad P=\sum_{i=1}^{N} F_{i} \Delta f_{i}^{1}, \\
& Q=\sum_{i=1}^{N} F_{i} \Delta f_{i}^{2}, \quad R=\sum_{i=1}^{N} F_{i} \Delta f_{i}^{3}, \\
& S=\sum_{i=1}^{N} F_{i} \Delta f_{i}^{4}, \quad T=\sum_{i=1}^{N} F_{i} \Delta f_{i}^{5}, \\
& U=\sum_{i=1}^{N} F_{i} \Delta f_{i}^{6}
\end{aligned}
$$

Coefficients $k_{0}$ to $k_{6}$ in (13) can be obtained, and the input and output variable regression of the SAW based micro force sensor can be calculated by solving (18).

\section{Testing and Analysis of the SAW Based Micro Force Sensor}

5.1. Actual Circuit Detection Results. The output frequency of the SAW based micro force sensor is conducted by using the network analyzer equipment E5061A (Figure 7). Forcemeasuring elements employ a cantilever beam loaded with $0-20 \mathrm{kPa}$ pressure and add $2 \mathrm{kPa}$ to this beam at each time [20].

Figure 8 shows the test schematic. The SAW based micro force sensor has been designed with a single channel. For the inductive components, the electromagnetic field interference is more sensitive; two inductors have been placed at $90^{\circ}$ to reduce interference between these components. Figure 9 shows the actual circuit. In the process of real production, the device location and the connection between our devices are more important. 
TABLE 1: Difference in frequency data for various pressures.

\begin{tabular}{|c|c|c|c|c|c|c|c|c|c|c|c|}
\hline \multirow[b]{2}{*}{$F_{m}$} & \multicolumn{11}{|c|}{$(\mathrm{kPa})$} \\
\hline & 0 & 2 & 4 & 6 & 8 & 10 & 12 & 14 & 16 & 18 & 20 \\
\hline$f(\mathrm{MHz})$ & 49.255389 & 49.255391 & 49.255378 & 49.255393 & 49.255434 & 49.255459 & 49.255474 & 49.255481 & 49.255447 & 49.255486 & 49.255497 \\
\hline$f(\mathrm{MHz})$ & 49.255321 & 49.255312 & 49.255298 & 49.255296 & 49.255338 & 49.255343 & 49.255357 & 49.255359 & 49.255319 & 49.255361 & 49.255363 \\
\hline$\Delta f(\mathrm{~Hz})$ & 68 & 79 & 80 & 97 & 96 & 116 & 117 & 122 & 128 & 125 & 134 \\
\hline
\end{tabular}

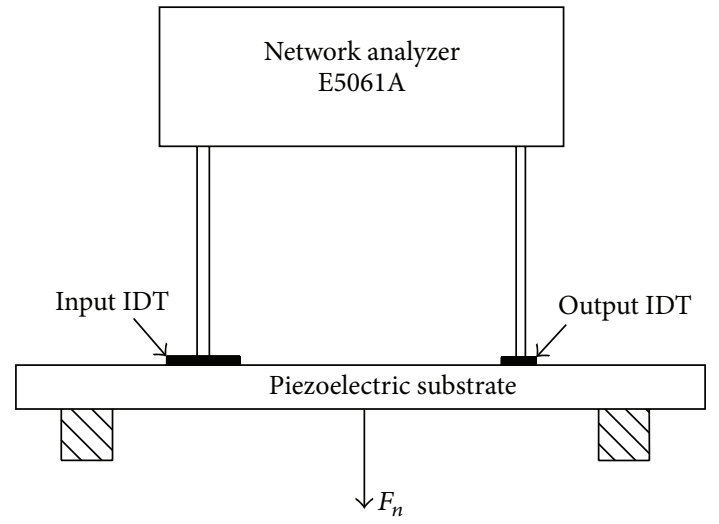

FIGURE 7: Schematic of the SAW based micro force sensor.

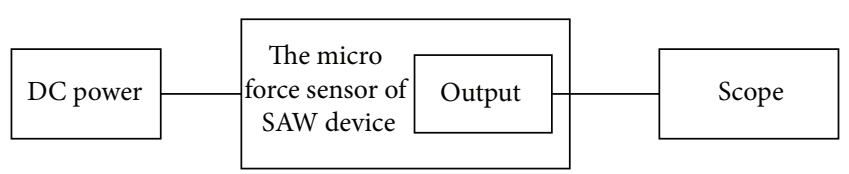

FIGURE 8: Test schematic diagram.

5.2. The Fitting Curve for the Measured Frequency. Figure 10 shows the actual test waveform diagram [21].

To eliminate the system instability, the experimental data has been averaged in Table 1 .

Frequency experiment data samples have been generated from (18) according to the different micro pressure reading, and the estimated regression coefficients $\hat{k}_{0}, \hat{k}_{1}, \hat{k}_{2}$, $\hat{k}_{3}, \hat{k}_{4}, \hat{k}_{5}$ and $\hat{k}_{6}$ can be calculated. The method of least squares can reduce the error due to measurement inaccuracies caused by the SAW device based micro force sensor. Through the minimization of squared errors of our actual data, the least squares method has the ability to find the best matching function data. Additionally, the fitting curve of the experimental data shows that the circuit design is reasonable and the device exhibits good linearity (Figure 11). The pressure $F_{m}$ is proportional to the output frequency $f$, which has proved that the SAW detection and pierce oscillator circuit have correct logic function.

Based on (13), the available in different micro force sensor output difference frequency transfer function is

$$
\begin{aligned}
F_{m}(\Delta f)= & 3.1441 \times 10^{-1}+5.9057 \times 10^{-2} \Delta f_{i} \\
& -2.3012 \times 10^{-4} \Delta f_{i}^{2}+5.4127 \times 10^{-7} \Delta f_{i}^{3}
\end{aligned}
$$

\begin{tabular}{|c|c|c|c|}
\hline Measurement parameter & $\begin{array}{l}\text { Average } \\
\text { value }\end{array}$ & $\begin{array}{l}\text { Minimum } \\
\text { value }\end{array}$ & $\begin{array}{l}\text { Maximum } \\
\text { value }\end{array}$ \\
\hline Voltage output (mV) & 320 & 314 & 326 \\
\hline $\begin{array}{l}\text { Frequency output } \\
(\mathrm{MHz})\end{array}$ & 49.2553965 & 49.255296 & 49.255497 \\
\hline
\end{tabular}

TABLE 2: Oscilloscope measurement results.

$$
\begin{aligned}
& -6.0364 \times 10^{-10} \Delta f_{i}^{4} \\
& +3.0200 \times 10^{-13} \Delta f_{i}^{5}-5.0540 \times 10^{-17} \Delta f_{i}^{6} .
\end{aligned}
$$

Because of the load impedance having affected the amplitude output of the micro force sensor, the impedance of the oscilloscope probe during testing is set at $1 \mathrm{~m} \Omega$ to stabilize the oscillator amplitude. The working circuit voltage and current are $3 \mathrm{~V}$ and $10 \mathrm{~mA}$. Using the difference in frequency data for various pressures in Table 1, the Oscilloscope measurement result has been calculated in Table 2 .

\section{Conclusion}

This paper has addressed three problems in designing the SAW based micro force sensor, namely, the envelope of IDT, the variable regression, and the fitting curve analysis for the measured frequency, which are according to the wavelet transform method, SAW detection, and pierce oscillator circuits.

The paper has also proposed the mixing frequency circuit for the reference oscillator and the detector circuit. The changes between the oscillator frequency and the measurement of the parameters can be obtained through the pierce oscillator circuits, and the difference in frequency data for various pressures also can be established by the linear regression model. By affecting the production of amplitude of impedance and parasitic capacitance, the output frequency of $50 \mathrm{MHz}$ still has stabilized features and performance. The parameters of frequency, amplitude, frequency stability, and amplitude stability have been measured during the device response and the sensor simulation.

According to the Morlet wavelet transform, SAW detection, and pierce oscillator circuits, the experimental results have confirmed that the SAW based micro force sensor can implement high reproducibility. When the input transducer of our device has been designed with the envelope of the conducting strips, our device performs well linearity. In addition, the device uses the piezoelectric properties and the temperature stability of the crystal, as well as the frequency 

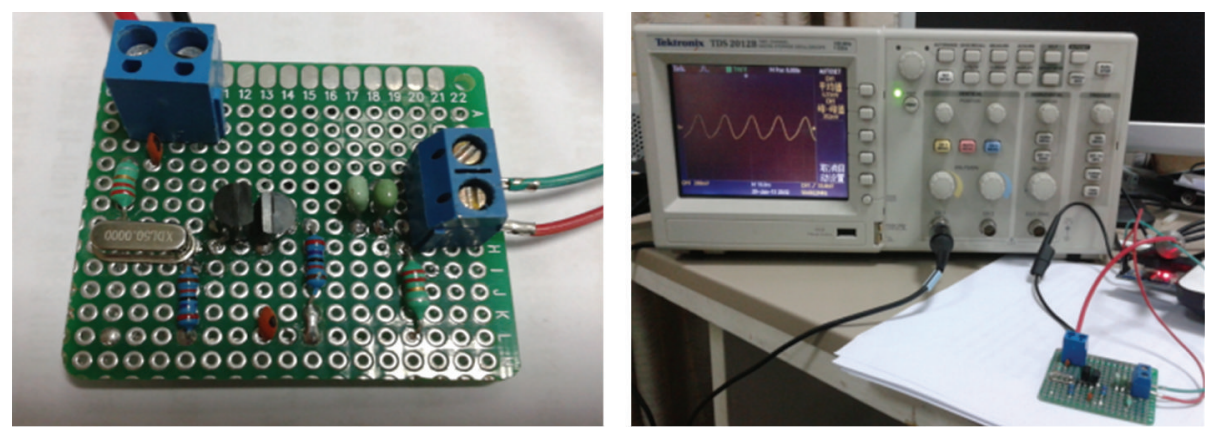

FIgURE 9: The actual circuit testing diagram.

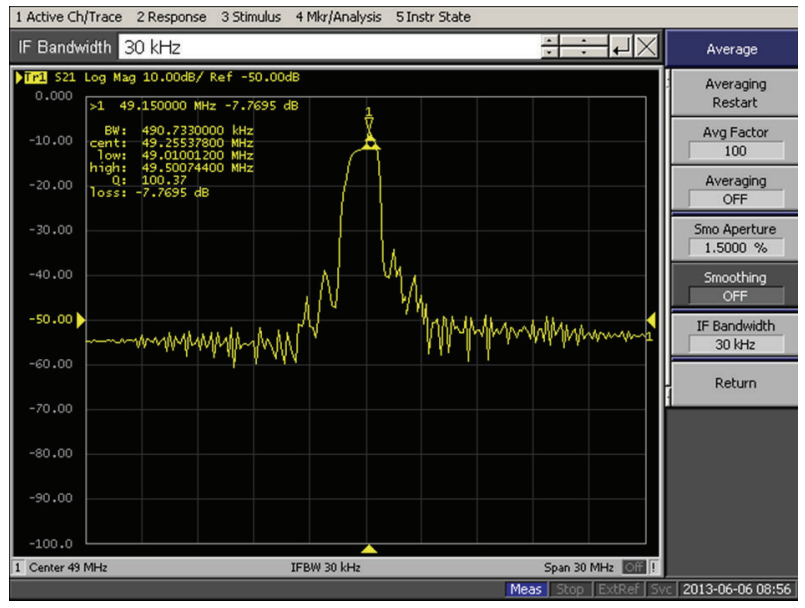

FIgURE 10: The actual test waveform diagram.

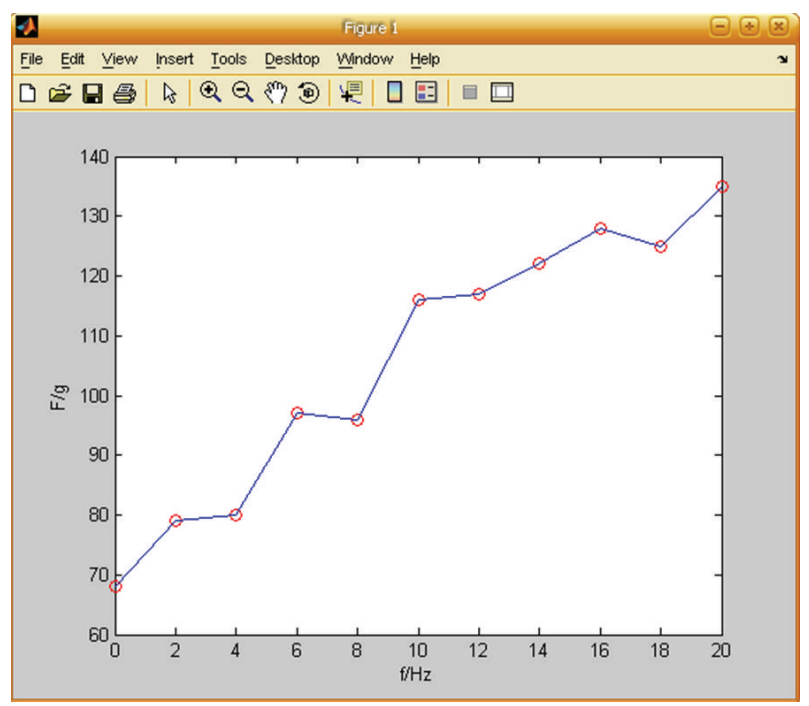

FIGURE 11: The experimental data fitting curve.

signal instead of the conventional pressure sensor with voltage signal, which has made the signal processing of this device more digital and possessed more stable performances. The SAW based micro force type can be fabricated and has high performance in either mobile micro robots or a precise positioning device under the control of mechanical system.

\section{Conflict of Interests}

The authors declare that there is no conflict of interests regarding the publication of this paper.

\section{Acknowledgment}

This work was supported by the National Natural Science Foundation of China (Grant no. 61274078), the Research Innovation and Project of the Shanghai Municipal Education Commission (Grant no. 13ZZ049), the Doctoral Scientific Fund Project of the Ministry of Education of China (Grant no. 20120075110006), and the Foundation of Shanghai University of Engineering Science (Grant no. nhky-2013-10).

\section{References}

[1] I. Daubechies, "The wavelet transform, time-frequency localization and signal analysis," IEEE Transactions on Information Theory, vol. 36, no. 5, pp. 961-1005, 1990.

[2] D. S. Ballantine, R. M. White, S. J. Martin et al., Acoustic Wave Sensor: Theory, Design, and Physico-Chemical Applications, Academic Press, New York, NY, USA, 1997.

[3] Y. H. Peng, Wavelet Transform and Its Engineering Application, Science Press, Beijing, China, 1999, (Chinese).

[4] W. K. Lu, C. C. Zhu, J. H. Liu, and Q. Liu, "Implementing wavelet transform with SAW elements," Science in China E: Technological Sciences, vol. 46, no. 6, pp. 627-638, 2003.

[5] W.-K. Lu, C.-C. Zhu, J.-H. Liu, and P.-Y. Wei, "Study on implementation of surface-acoustic-wave type of the wavelettransformation and reconstruction element," Acta Electronica Sinica, vol. 30, no. 8, pp. 1156-1159, 2002 (Chinese).

[6] K. Andra, C. Chakrabarti, and T. Acharya, "A VLSI architecture for lifting-based forward and inverse wavelet transform," IEEE Transactions on Signal Processing, vol. 50, no. 4, pp. 966-977, 2002.

[7] K. K. Parhi and T. Nishitani, "VLSI architecture for discrete wavelet transforms," IEEE Transactions on Very Large Scale Integration (VLSI) Systems, vol. 1, no. 2, pp. 191-202, 1993.

[8] X. Chen, X. Zhang, K. Chen, and Q. Li, "Optical waveletmatched filtering with bacteriorhodopsin films," Applied Optics, vol. 36, no. 32, pp. 8413-8416, 1997. 
[9] M. von Schickfus, R. Stanzel, T. Kammereck, D. Weiskat, W. Dittrich, and H. Fuchs, "Improving the SAW gas sensor: device, electronics and sensor layer," Sensors and Actuators B: Chemical, vol. 19, no. 1-3, pp. 443-447, 1994.

[10] M. K. Tan, L. Y. Yeo, and J. R. Friend, "Rapid fluid flow and mixing induced in microchannels using surface acoustic waves," Europhysics Letters, vol. 87, no. 4, pp. 537-563, 2009.

[11] W. C. Wilson, D. C. Malocha, N. Y. Kozlovski et al., "Orthogonal frequency coded SAW sensors for aerospace SHM applications," Sensors Journal, vol. 9, no. 11, pp. 1546-1556, 2009.

[12] M. Jungwirth, H. Scherr, and R. Weigel, "Micromechanical precision pressure sensor incorporating SAW delay lines," Acta Mechanica, vol. 158, no. 3-4, pp. 227-252, 2002.

[13] S. Muntwyler, F. Beyeler, and B. J. Nelson, “Three-axis microforce sensor with sub-micro-Newton measurement uncertainty and tunable force range," Journal of Micromechanics and Microengineering, vol. 20, no. 2, pp. 3165-3170, 2010.

[14] Y. C. Kim, Y. S. Ihn, H. Moon et al., "Low cost dual axis micro force sensor for robotic manipulations," Microsystem Technologies, vol. 17, no. 5-7, pp. 1197-1205, 2011.

[15] K. J. Singh, O. Elmazria, F. Sarry et al., "Enhanced sensitivity of SAW-based Pirani vacuum pressure sensor," IEEE Sensors Journal, vol. 11, no. 6, pp. 1458-1464, 2011.

[16] C. B. Wen and C. C. Zhu, "Time synchronous dyadic wavelet processor array using surface acoustic wave devices," Smart Materials and Structures, vol. 15, no. 4, pp. 939-945, 2006.

[17] W. K. Lu, C. C. Zhu, J. F. Zhang, C. Shi, and X. Z. Lü, "Study of small size wavelet transform processor and wavelet inversetransform processor using SAW devices," Measurement: Journal of the International Measurement Confederation, vol. 44, no. 5, pp. 994-999, 2011.

[18] Y. Kang, Design of Surface Acoustic Wave Devices and Its Application Oscillator Circuit, Chang'an University, 2011.

[19] X. Z. Lü, Interfacial Stress Sensor for Artificial Skin Application, Donghua University, 2012.

[20] Y. Y. Li, W. K. Lu, C. C. Zhu et al., "Acoustic electric generation for morlet wavelet transform of surface acoustic wave device," Research Journal of Applied Sciences, Engineering and Technology, vol. 5, no. 4, pp. 1203-1207, 2013.

[21] Y. Y. Li, W. K. Lu, and C. C. Zhu, "Pspice equivalent circuit model for implementation of surface acoustic wave filter," Journal of Donghua University, vol. 29, no. 2, pp. 148-152, 2012. 


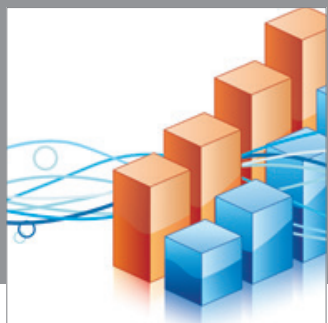

Advances in

Operations Research

mansans

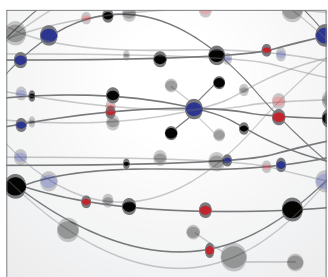

The Scientific World Journal
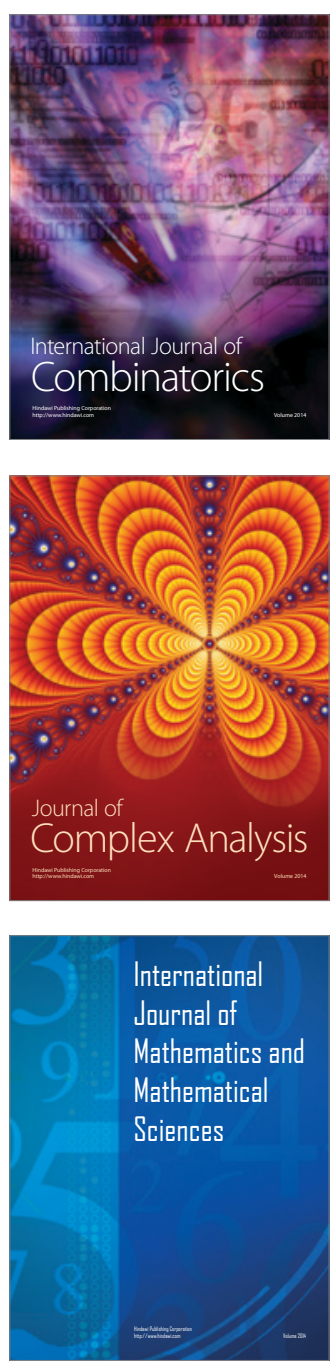
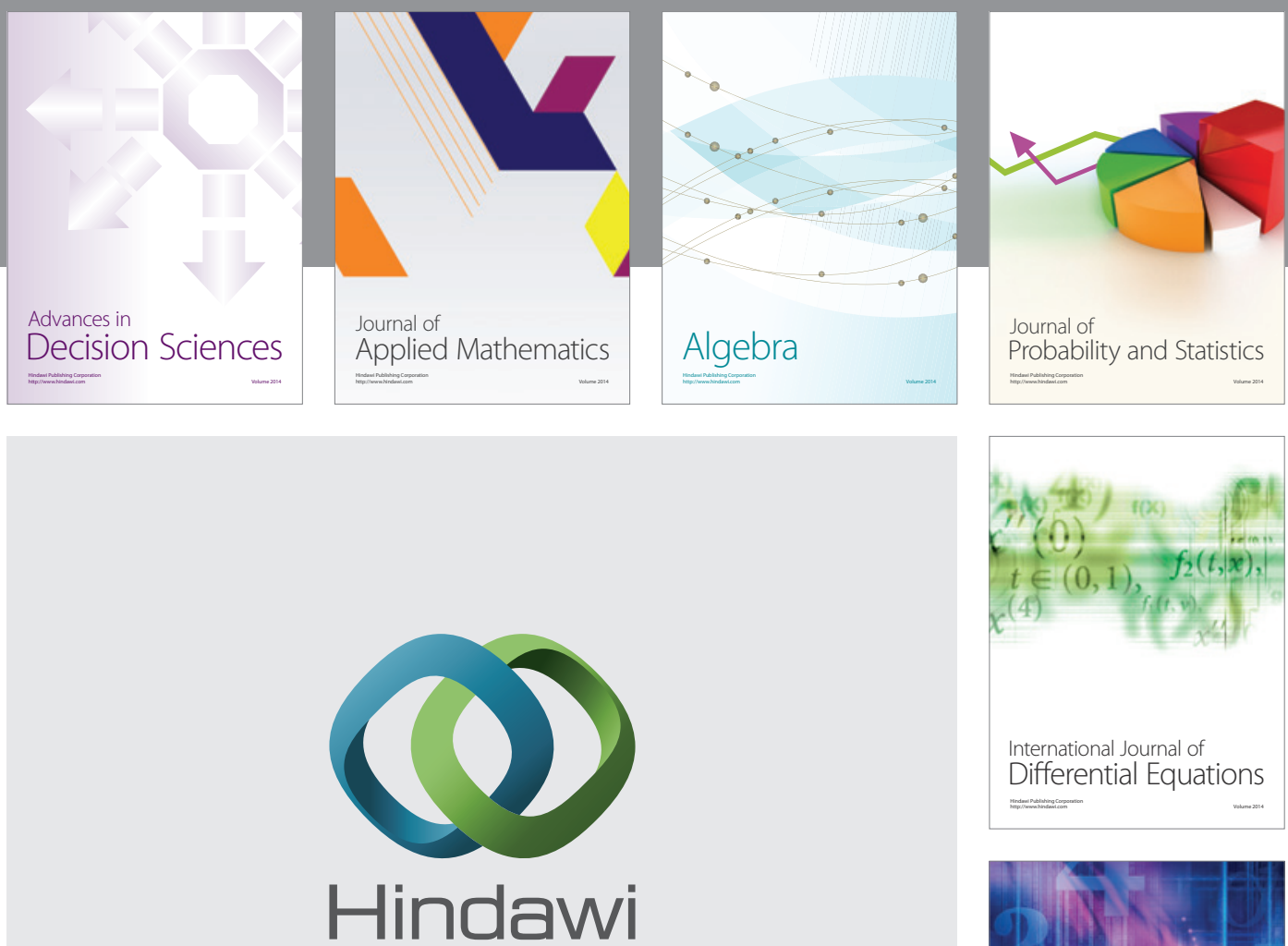

Submit your manuscripts at http://www.hindawi.com
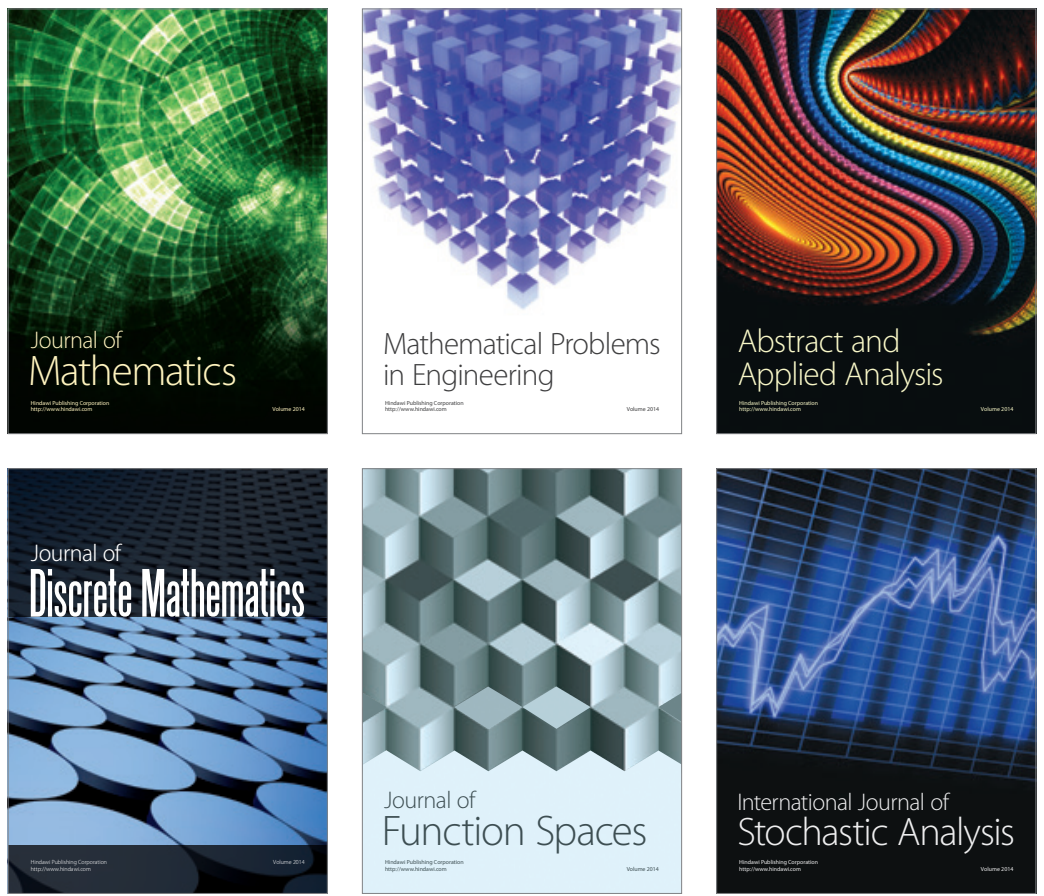

Journal of

Function Spaces

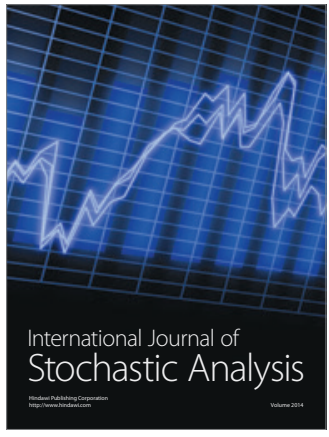

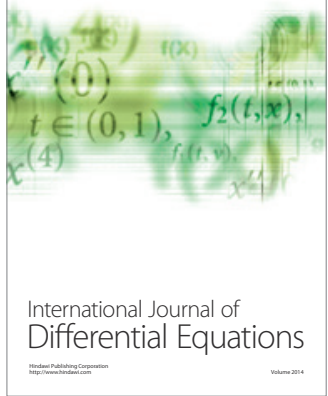
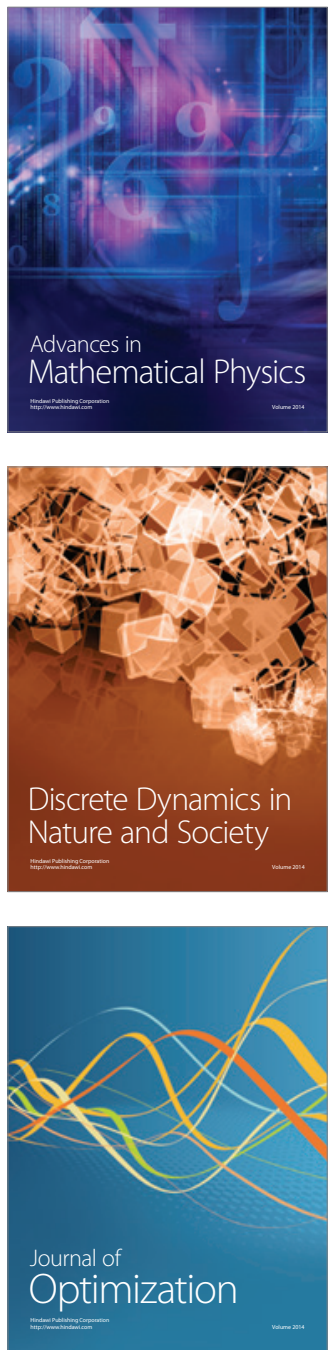